\title{
FOREWORD
}

\section{Amphibian chytridiomycosis}

\author{
Alex D. Hyatt ${ }^{1, *}$, Richard Speare ${ }^{2}$, Andrew A. Cunningham ${ }^{3}$, Cynthia Carey ${ }^{4}$ \\ ${ }^{1}$ CSIRO, Livestock Industries, Australian Animal Health Laboratory, Geelong, Victoria 3220, Australia \\ ${ }^{2}$ James Cook University, Townsville, Queensland 4811, Australia \\ ${ }^{3}$ Institute of Zoology, Regent's Park, London NW1 4RY, UK \\ ${ }^{4}$ Department of Integrative Physiology, University of Colorado, Boulder, Colorado 80309-0354, USA
}

\begin{abstract}
Amphibian chytridiomycosis is a fungal disease caused by the chytrid fungus Batrachochytrium dendrobatidis. It is arguably the most significant recorded infectious disease of any vertebrate class. The disease is reducing amphibian biodiversity across most continents and regions of the world, affecting the resilience of surviving populations and driving multiple species to extinction. It is now recognised by the World Organisation for Animal Health (OIE) as an internationally notifiable disease. Collaborative research in areas including the development of diagnostic assays, distribution and impact of the disease, and management (treatment and policy) has assisted in leading a paradigm shift in accepting infectious disease as a major factor influencing wildlife population stability and biodiversity.
\end{abstract}

KEY WORDS: Chytridiomycosis $\cdot$ Batrachochytrium dendrobatidis $\cdot$ Amphibian $\cdot$ Fungus $\cdot$ Chytrid

This DAO Special focusses on a pathogenic fungus that is causing a pandemic among many species of amphibians on all 6 continents on which they occur (Antarctica lacks amphibian hosts). The pandemic has resulted in the most spectacular loss of biodiversity in recorded history.

Amphibian chytridiomycosis is a fungal disease which was unknown until it was first described in 1998 and is now recognised to be pandemic. It is a major research focus; the collection of papers published here illustrate its importance. Fungal diseases rarely cause serious epidemics with high mortality rates in vertebrates, and amphibian chytridiomycosis is arguably unique in becoming a pandemic, infecting and killing amphibians globally. The disease is caused by the chytrid fungus, Batrachochytrium dendrobatidis (to the horror of formal taxonomists the abbreviation ' $B d$ ' is frequently used). Since it has an infective aquatic stage, it is associated with fresh water bodies. The original name used for the disease, cutaneous chytridiomycosis, is more informative (although the full name is now rarely used) because the fungus does not invade deep into the body, but remains confined to keratinised structures, namely the skin. In larval anurans, $B d$ invades the keratinised structures in the mouth and, as the tadpole approaches metamorphosis, $B d$ infects the keratinised epithelium as it develops. In metamorphosed amphibians, the surface layers of the skin (the stratum corneum and the stratum granulosum) are invaded. The infected epidermis is damaged; it proliferates to become thicker and frequently sloughs prematurely. The adaptive immune response is minimal, suggesting that either the amphibian host does not immunologically 'see' the fungus or that $B d$ is able to suppress the host's immune response. Perhaps there may be pharmacological spin-offs from this terrible disease if the latter is the case!

The superficial and localised nature of the infection delayed its discovery as, even when disease investigations occurred, the traditional examination of viscera failed to identify a cause of death. But how can a very superficial infection cause mortality? The answer to this question was elusive for many years, even though experimental evidence proved the virulence of $B d$. 
Evidence now shows that infection with the fungus interferes with the sodium-potassium pump in the epidermis and, if infection is extensive, electrolytes leak out of the circulation, $\mathrm{pH}$ falls and the heart rate slows and finally stops. Death is not due to a pathogen swarming through tissues, but to a superficial skin infection initiating a metabolic imbalance that elegantly disrupts the function of a vital organ. Although we have had strategies for saving infected frogs in captivity for many years with the use of antifungal agents, discovery of the mechanism that leads to death has opened up further possibilities for saving severely affected amphibians by correcting the metabolic abnormality to buy time to allow antifungal therapy to work. This is particularly important since captivity is currently the only saviour for amphibian species threatened with immediate extinction by $B d$ in the wild.

$B d$ is exquisitely sensitive to antifungal agents, usually dying in vitro at much lower concentrations than other fungi. In addition, it is exquisitely sensitive to heat, dying above $32^{\circ} \mathrm{C}$. Even contact with human skin can kill Bd! Hence hygiene protocols are well developed and non-organic components of captive environments can be kept free of $B d$. Attempts at killing $B d$ in the amphibian host, however, have had variable results. The fungus is possibly protected in its intracellular location within the keratinised epithelium. To date only one treatment has been found to be effective at eliminating $B d$, itraconazole. Other treatments, such as heating regimes using temperatures of $37^{\circ} \mathrm{C}$ for tropical frogs and $32^{\circ} \mathrm{C}$ for temperate frogs, offer further promise for a physical control strategy. The lack of pharmacological background data on antifungal drugs in amphibian hosts is a major gap in our knowledge. Antifungal therapy for chytridiomycosis will not advance until basic studies are conducted on pharmacokinetics and randomised controlled trials are used. Proposals to control chytridiomycosis in wild amphibian populations using antifungal agents might make theoretical sense, but face huge challenges, including the need for better data on the benefits and adverse effects of targeted applications of licensed agricultural antifungals. Therapy and control of chytridiomycosis in the wild using antifungals has great potential, but needs much more research effort.

$B d$ has been moved around the world in infected amphibians traded as pets, food and laboratory animals for scientific research. The inadvertent movement of 'stow-away' amphibians in produce and other goods also has been identified as a likely method for the international spread of $B d$. How $B d$ moves from habitat to habitat is still an area of speculation; infected animals can move the pathogen from pond to pond, but other natural mechanisms, such as mechanical trans- port via birds or aquatic insects, have not been ruled out. Since chytridiomycosis is an emerging infectious disease that has a highly significant impact on global amphibian populations, the movement of amphibians is now becoming subject to more stringent and specific conditions. The World Organisation for Animal Health (OIE) has made chytridiomycosis an internationally notifiable disease and countries should adhere to tighter protocols when exporting amphibians. OIE validated testing protocols for the detection of $B d$ have been written and will soon be published in the OIE 'Manual of Diagnostic Tests for Aquatic Animals'.

When $B d$ first arrives in a susceptible population it can cause high rates of mortality as it sweeps through the countryside on an epidemic wave. Once the initial wave has passed, $B d$ becomes endemic in the remaining amphibian populations, with species that are less susceptible to amphibian chytridiomycosis acting as reservoirs of infection for those that are highly susceptible to the disease. Overt, direct mortality is not the only outcome of infection, however, as infected animals can exhibit reduced fitness and surviving populations infected with $B d$ can have reduced resilience to stochastic events. Climatic and other environmental conditions can interact with amphibians and $B d$ to influence the impact of infection. In tropical areas, mortality rates tend to be greater where temperatures are cooler, so mortality rates often are most spectacular in upland regions; in lowlands the higher temperatures can be protective, reducing mortality rates even for susceptible species. For highly susceptible species localised to small areas and living in environments with conditions that favour $B d$, extinction can occur. This can be rapid (<1 yr) (e.g. golden toad, sharpsnouted torrent frog) and associated with the initial invasion of the pathogen. For some species (e.g. the southern corroboree frog), extinction might not be a spectacular 'there one day gone the next', but realised as a slow, steady decline over years. Multiple extinction events have been seen, particularly in Australia and Central America. Globally, however, the major impact of chytridiomycosis may be on reducing fitness of tadpoles, juveniles and adults in surviving populations. Sophisticated research is currently investigating this more subtle impact, the effects of which will become more apparent over time.

What is being done to decrease the impact of chytridiomycosis and stop loss of amphibian biodiversity? At the global level we have OIE and an international legislative approach requiring countries to recognise the problem and take steps to reduce the international spread of $B d$. Even if $B d$ is present in a country, different fungal strains vary in virulence, so limiting the strain complement in a country is important. However, for wildlife managers and policy mak- 
ers to take notice of strain differences, better evidence of differential impact in wild amphibian populations may be needed. At the national level, various countries are developing national plans to control chytridiomycosis. Emphasis is placed on implementing hygiene protocols for individuals dealing with wild amphibians and recognising that pristine, isolated areas containing highly vulnerable species need to be protected from $B d$. Time is being bought through establishing captive populations for highly susceptible species threatened by advancing epidemic waves (the Amphibian Ark project) or already infected populations suffering slow, steady declines (southern corroboree frog), but captivity is a stop-gap and not a solution. Even if all zoos in the world cooperated - and such cooperation seems to still be a distant dream - it is estimated that only 50 of the 500 species in imminent danger of extinction could be saved in this way. Also, these programmes are longterm undertakings with no clear exit strategy: $B d$ is not known to have been lost from any area it has reached. Clearly, if catastrophic losses of amphibian species due to chytridiomycosis are to be averted, alternative mitigation techniques need to be developed and this remains one of the greatest challenges to amphibian conservation worldwide. Rising to this challenge include projects investigating better therapies for infected frogs, gaining understandings of the amphibian immune response to $B d$ (is a vaccine possible?), researching in situ treatment strategies, and investigating if frogs that survive after a chytridiomycosis epidemic have been selected for resistance and could pos- sibly form breeding nuclei to establish $B d$-resistant wild populations.

Amphibian chytridiomycosis is a devastating disease. On a global scale it is, arguably, the most significant infectious disease of any vertebrate class, ever. It is decreasing amphibian biodiversity across most continents and regions of the world, affecting the resilience of surviving populations and driving multiple species to extinction. We are only just waking up to the ecological consequences of such destruction of a key cog in the wheel of ecosystem function. Recognition of the impact of this disease has, however, helped lead a paradigm shift in the field of ecology, opening up acceptance of the theory of infectious disease as a major factor influencing wildlife population stability and biodiversity conservation. Amphibian chytridiomycosis has allowed wildlife managers to understand how important infectious diseases are to wildlife management. It has provided the opportunity to seriously consider how and when a disease of wildlife can and should be controlled. Much more research needs to be done and we hope this DAO Special helps to provide additional evidence to allow us to rise to the challenge and beat this global threat.

The papers published here are representative of the large on-going area of research focusing on identifying the global distribution of $B d$, modification of diagnostic assays, increasing our understanding of the biology and genetics of the pathogen and, ultimately, in improving our abilities to control the disease. 